\title{
Bilateral sequential theta burst stimulation in depressed veterans with service related posttraumatic stress disorder: a feasibility study
}

\author{
Thelepa Vaithianathan ${ }^{1}$, Mervin Blair ${ }^{1,2}$, Vanessa Soares ${ }^{2,3}$, Yuri E. Rybak ${ }^{4}$, Lena Palaniyappan ${ }^{4,5}$,
} J Don Richardson ${ }^{2,3,4}$ and Amer M. Burhan 1,4,6*

\begin{abstract}
Background: Depression comorbid with posttraumatic stress disorder (PTSD) can be disabling and treatment resistant. Preliminary evidence suggests that repetitive transcranial magnetic stimulation (rTMS), may have a role in helping these patients. There are only few published studies using different rTMS paradigms including bilateral intermittent theta burst (iTBS) and low frequency rTMS.

Methods: In this small cohort observation study, we examined the efficacy of bilateral sequential theta-burst stimulation (bsTBS) in 8 treatment resistant depression (TRD) military veterans with PTSD comorbidity stemming from military service experience.

Results: bsTBS was generally well tolerated and resulted in 25\% and 38\% remission and response rates on Depression scores respectively; $25 \%$ remission and response rate on PTSD scores.

Discussion: This study demonstrates preliminary feasibility and safety of bsTBS in TRD with comorbid military service related PTSD.

We concluded that this paradigm might hold promise as a therapeutic tool to help patients with TRD co-morbid with military service related PTSD. Further adequately powered studies to compare rTMS treatment paradigms in this patient group are warranted.
\end{abstract}

Keywords: TRD, PTSD, theta burst stimulation, Military, Veterans

\section{Introduction}

Posttraumatic stress disorder (PTSD) can be comorbid with other illnesses and can cause significant impairment [1]. Current interventions for PTSD are limited in efficacy [2]. Consequently, there is a need for new interventions.

Repetitive transcranial magnetic stimulation (rTMS) is a relatively non-invasive procedure increasingly used to treat psychiatric disorders. A meta-analysis of 17 studies

${ }^{*}$ Correspondence: burhana@ontarioshores.ca

${ }^{1}$ Ontario Shores Centre for Mental Health Sciences, 700 Gordon Street,

Whitby, Ontario L1N 9X4, Canada

Full list of author information is available at the end of the article demonstrated that right dorsolateral prefrontal cortex (DLPFC) high frequency stimulation is the most effective treatment for PTSD [3]. Standard high frequency rTMS requires $37.5 \mathrm{~min}$ to deliver. Intermittent theta burst stimulation (iTBS) can be delivered in 3 min and is noninferior to high frequency rTMS in treating treatment resistant depression (TRD) [4].

Two studies examined iTBS in PTSD and showed a reduction in depression and PTSD symptoms $[5,6]$. The site of stimulation differed between the two studies (right iTBS and bilateral iTBS respectively). A recent study by Philip et al. [7] showed that rTMS, at 5-Hz, was superior to iTBS in the treatment of PTSD symptoms when 
both are applied to the left DLPFC only. To treat PTSD, a meta-analysis by Kan et al. [8] supported right-sided inhibitory protocols, although at somewhat lower effectsize than left-sided DLPFC stimulation.

For depression, especially TRD, several lines of evidence support the use of left DLPFC stimulation $[9,10]$. Evidence suggests that patients with depressive disorders present with frontal activity asymmetry (right $>$ left) [11, 12]. While this still needs to be clarified further given the heterogeneity of studies, rTMS studies utilizing right inhibitory stimulation followed by left excitatory stimulation have shown to be effective in TRD $[9,10,13,14]$. Thus, in the present retrospective study in which bilateral stimulation was applied, we reasoned that inhibitory protocol (cTBS) on the right DLPFC would alleviate PTSD symptoms, while left-sided excitatory protocol (iTBS) would help TRD symptoms. With respect to burst sequence, Plewnia et al. [15] found that the bilateral TBS protocol, that we used, was safe and superior to sham stimulation for the treatment of major depression. Further, we recently reported good efficacy and tolerability for bilateral sequential theta-burst applications in TRD [16].

In the current retrospective chart review, we examined the efficacy and safety of bilateral sequential TBS (bsTBS) in patients with TRD and co-morbid PTSD. We investigated the notion that right sided cTBS would improve PTSD symptoms and left sided iTBS would reduce depressive symptoms $[17,18]$.

\section{Methods}

This is a retrospective chart review and was conducted in accordance with the Declaration of Helsinki and was approved by the Research Ethics Board at the University of Western Ontario (project ID: 116,274). Data was collected retrospectively on 15 TMS clinic patients referred for TRD and PTSD, as such, informed consent was not required for data collection, however was obtained prior to treatment. The data sets used during the current study are available from the corresponding author upon request.

\section{Inclusion}

18 years and older, diagnosed with TRD and PTSD and received a minimum of 10 bsTBS sessions.

The patients were referred from the Operational Stress Injury Clinic, having the diagnosis of military related PTSD. The diagnosis of PTSD was made by a psychiatrist through a comprehensive clinical assessment in combination with the Clinical Administered PTSD Scale (CAPS) and the self-administered PTSD checklist (PCL5). The diagnosis of TRD was obtained by using clinical impression of a major depressive episode with partial or no response to at least two optimum courses of antidepressant trials per referring psychiatrist and as confirmed on intake by the Therapeutic Brain Stimulation Clinic.

\section{Exclusion}

3 received different rTMS paradigms and 4 patients withdrew (3 due to side effects including headaches and 1 due to scheduling conflicts).

Eight patients received 19 to 30 sessions over $4-6$ weeks.

Baseline and post bsTBS assessments were obtained from the HAMD-17, Generalized Anxiety Disorder Scale (GAD-7), Patient Health Questionnaire (PHQ-9), PTSD Checklist for DSM-5 (PCL-5), and Clinical Global Impression Severity-Improvement Scale (CGI-S, CGI-I).

The primary outcome was the change in HAMD-17 score from baseline to the end of treatment. Secondary outcomes included change in PCL-5, PHQ-9, GAD-7 and CGI scores. Clinical response was defined as $>=50 \%$ reduction of baseline scores on the HAMD-17, PCL-5, PHQ-9 and GAD-7, whereas remission was defined by HAMD-17 score of $<=7$ [19]. We opted to define remission for the remaining scales when scores fell below the normative cut-offs: PCL-5 score of $<31-33$ [20], PHQ-9 score of $<5$ [21] and GAD-7 score of $<5$ [22].

The TMS Machine (Magstim ${ }^{\circledR}$ Rapid 2, UK) was used to apply the paradigm published previously [16]. Briefly, 600 pulses of continuous TBS (cTBS) was applied to F4 location and subsequently iTBS to F3 location with burst frequency between 40 and $50 \mathrm{~Hz}$. We used Beam F3 method to identify F3 location on the left side [23] and the corresponding F4 location on the right side. Resting motor threshold (RMT) was determined by eliciting a visible muscle twitch in the thumb or index finger on the right hand in 3 of 5 stimulation trials. Energy between 80 and $120 \%$ of the RMT was used depending on tolerability.

\section{Results}

Eight male patients between ages 30 and 60 were included. All 8 patients received combination $(2+$ antidepressants) or augmentation (antidepressant plus nonantidepressant) treatment during their lifetime and were receiving medication for depression at time of treatment. The demographics of each patient with number of sessions, stimulus parameters and change in severity of illness is presented in Table 1.

Following bsTBS, 38\% and 25\% met response and remission criteria respectively based on HAMD-17 scores. Results were comparable based on self-rating for PCL-5 (25\% response and remission), GAD-7 (38\% and 25\% respectively), and PHQ-9 (25\% and 13\% respectively). 
Table 1 Demographics and parameters of bilateral rTMS treatments received by participants

\begin{tabular}{|c|c|c|c|c|c|c|c|c|c|c|}
\hline \multirow[b]{2}{*}{ ID } & \multirow{2}{*}{$\begin{array}{l}\text { Demographics } \\
\text { Age (sex) }\end{array}$} & \multicolumn{4}{|c|}{ Parameters } & \multicolumn{4}{|c|}{ Rating scales (Pre/Post) } & \multirow{2}{*}{$\begin{array}{l}\text { Clinical } \\
\text { Impression } \\
\text { CGI } \\
\text { (S/C) }\end{array}$} \\
\hline & & \%RMT & Left (F3) & Right (F4) & Sessions & HAMD-17 & PHQ-9 & GAD-7 & PCL-5 & \\
\hline 1 & $59(\mathrm{M})$ & 80 & iTBS & CTBS & 20 & $16 / 17$ & $22 / 21$ & 18/18 & - & $-/ 3$ \\
\hline 2 & $53(\mathrm{M})$ & 100 & iTBS & CTBS & 20 & $25 / 17$ & $23 / 18$ & $18 / 15$ & $-/$ & $5 / 3$ \\
\hline 3 & $56(M)$ & 100 & iTBS & CTBS & 30 & $18 / 12$ & $23 / 13$ & $18 / 9$ & $42 / 40$ & $4 / 2$ \\
\hline 4 & $39(\mathrm{M})$ & 100 & iTBS & CTBS & 27 & 29/11 & $19 / 16$ & $16 / 12$ & $62 / 47$ & $6 / 3$ \\
\hline 5 & $50(\mathrm{M})$ & 100 & iTBS & CTBS & 19 & $23 / 3$ & $13 / 2$ & $8 / 0$ & $45 / 6$ & $6 / 1$ \\
\hline 6 & $56(M)$ & 80 & iTBS & CTBS & 29 & $26 / 7$ & $17 / 6$ & $14 / 3$ & $51 / 21$ & $5 / 1$ \\
\hline 7 & $43(M)$ & 100 & iTBS & cTBS & 30 & $21 / 20$ & $17 / 17$ & $14 / 10$ & $50 / 41$ & $5 / 4$ \\
\hline 8 & $34(M)$ & 100 & iTBS & cTBS & 30 & $26 / 16$ & $17 / 10$ & $11 / 7$ & 48/- & $5 / 2$ \\
\hline
\end{tabular}

Note: Scores for pre-treatment and post-treatment indicate changes to psychiatric scale scores showing changes due to treatment. Severity of illness is scored using the Clinical Global Impression (CGI) scale. CGI-S indicates the score given by the physician, ranging from 1 (normal, not at all ill) to 7 (among the most extremely ill patients in the population). The CGI-C indicates the change in CGI score after the treatment. Scoring for this scale ranges from 1 (very much improved) to 7 (very much worse)

iTBS intermittent theta-burst stimulation delivered at $3 \mathrm{~s}$ trains of $3 \times 50 \mathrm{hz}$ bursts at $5 \mathrm{hz}$ burst frequency with 8 inter-train seconds delay, 67 trains $=603$ pulses total CTBS Continuous train of $200 \times 3 \times 50 \mathrm{hz}$ bursts at $5 \mathrm{hz}$ burst frequency, with no intertrain delay $=600$ pulses total

Pre- to post-treatment improvement was evident for HAMD-17 (44\%; Cohen's d = 1.325), PCL-5 (37.59\%; Cohen's d = 1.370), PHQ-9 (31.78\%; Cohen's d = 1.445) and GAD-7 (36.78\%; Cohen's d = 1.586) (Fig. 1).

The average CGI-S score at baseline was 5.14 (SD = $0.64)$, (range from markedly ill to severely ill). The average change in CGI-I score at post-treatment was 2.38 (SD = 0.99) (range from minimally improved to much improved).

Most patients experienced mild side effects (muscle contraction, pain/discomfort, scalp irritation and changes in systolic blood pressure). No patient experienced syncope or seizures.

Three patients returned for additional bsTBS after 4 , 9 and 17 months, $2 / 3$ of patients responded again to the same paradigm.

\section{Discussion}

In this retrospective study, we examined the efficacy and safety of bsTBS in 8 patients referred to a TMS clinic for TRD comorbid with PTSD. The treatment was well




tolerated and resulted in improvement in depressive, anxiety and PTSD symptoms. We chose this paradigm given the evidence of right-left activity asymmetry in depression. In a recent review Bruder et al. [12], outlined the evidence from multiple sources indicating relative hypoactivity of left DLPFC in depression especially in the presence of anxiety symptoms and in relation of the failure to inhibit the right amygdala in response to emotional stimuli. We speculated that using inhibitory TBS on right DLPFC followed by excitatory TBS on the left DLPFC would result in improvement of depressive and anxiety symptoms in our cohort.

Further support for the right DLPFC target in PTSD comes from the largest sham-controlled study of its kind including 50 PTSD patients, whereby Philip et al. [5] applied iTBS to right DLPFC targeting PTSD symptoms, and found a small effect size on PCL-5 after two weeks of treatment (Cohen's $\mathrm{d}=0.34$ ).

Two recent papers published sub-sequent to our study, supported that right DLPFC is a good target to treat PTSD symptoms regardless if it is excitatory or inhibitory (McGirr [24], Khan [8]). There is evidence to suggest that bilateral rTMS might be superior to unilateral high frequency rTMS in remission rate in TRD $[13,14]$. There is paucity of studies that used bilateral TBS to treat depression or PTSD.

On the other hand, a small observation study similar to ours, including 8 PTSD patients (7 males) [6], utilized bilateral iTBS and observed a treatment effect on both PTSD (clinician-administered PTSD Scale for DSM5, CAPS-5, clinician rated structured interview, Cohen's d $=1.78$ ) and depressive symptoms (HAMD-17 Cohen's d $=1.16$ ). Our sample showed a slightly higher effect size on depression ( HAMD-17 Cohen's $d=1.33$ ). In our study effect size on self-rated PTSD symptoms was moderate (PCL-5 Cohen's $d=1.4)$. The latter is higher than the effect size reported in Philip et al. [5], which was a controlled blinded study reducing placebo effect, and lower than that reported in Nursey et al. [6], which used a clinician administered rating scale limiting the validity of the comparison.

Limitation of our study include: small sample size, only males, and being uncontrolled. Our iTBS paradigm, while it is designed using the same physiological principles, differed from the published iTBS paradigm.

Taken together, this study points out to the need for a larger, controlled studies for TRD co-morbid with PTSD. Furthermore, comparing paradigms that has promise in this population including right sided versus bilateral sequential, utilizing different combination of iTBS and cTBS, would inform the field about the most efficient method to treat TRD in PTSD.

\section{Acknowledgements}

AMB would like to acknowledge the in-kind support of the Therapeutic Brain Stimulation Clinic at Parkwood Institute-Mental Health Care in London, Ontario, CANADA.

\section{Authors' contributions}

$T V$ and $M B$ : wrote the main manuscript text under the supervision of AMB. AMB, YER and DJR: designed the treatment paradigm, recruited patients and provided treatment and assessment data, edited the manuscript. LP: provided feedback and edited the manuscript. VS: did the statistical analysis, prepared the figure, and provided edits on the manuscript. The author(s) read and approved the final manuscript.

\section{Funding}

Not applicable.

\section{Availability of data and materials}

Data set used in this study is available from corresponding author upon proper request.

\section{Declarations}

\section{Ethics approval and consent to participate}

This is a retrospective chart review so informed consent was not required for data collection for this research study. However following local hospital processes, consent was obtained from patients by treating psychiatrists prior to clinical treatment in the TMS clinic. This study was conducted in accordance with the Declaration of Helsinki and was approved by the Research Ethics Board at the University of Western Ontario (project ID: 116274).

\section{Consent for publication}

All authors have consented for the publication on this report. Informed consent for publication of aggregate data from patients is not applicable given that this is a retrospective chart review study approved as such by Research Ethics Board at the University of Western Ontario (project ID: 116274).

\section{Competing interests}

None for this work.LP reports personal fees from Janssen Canada, Otsuka Canada, SPMM Course Limited, UK, Canadian Psychiatric Association; book royalties from Oxford University Press; investigator-initiated educational grants from Janssen Canada, Sunovion and Otsuka Canada outside the submitted work. LP received travel support as a speaker for a meeting at Oxford, UK organized by Magstim limited in 2014. None related to this work. AMB reports personal fees from Janssen Canada, Avanir and Eisai pharmaceuticals for serving on advisory boards and from Atheneum Partners for providing expert consultation on market assessment. None are related to this work.

\section{Author details}

${ }^{1}$ Ontario Shores Centre for Mental Health Sciences, 700 Gordon Street, Whitby, Ontario L1N 9X4, Canada. ${ }^{2}$ Lawson Health Research Institute, London, Ontario, Canada. ${ }^{3}$ MacDonald Franklin OSI Research Centre, London, Ontario, Canada. ${ }^{4}$ Department of Psychiatry, Schulich School of Medicine and Dentistry, University of Western Ontario, Ontario, London, Canada. ${ }^{5}$ Robarts Research Institute, University of Western Ontario, London, Ontario, Canada. ${ }^{6}$ Department of Psychiatry, Temerty Faculty of Medicine, University of Toronto, Toronto, Ontario, Canada.

Received: 10 March 2021 Accepted: 19 January 2022

Published online: 03 February 2022

\section{References}

1. Breslau N. The epidemiology of posttraumatic stress disorder: what is the extent of the problem? J Clinical Psychiatry. 2021;62(Suppl 17):16-22.

2. Astill Wright L, Sijbrandij M, Sinnerton R, Lewis C, Roberts NP, Bisson Jl. Pharmacological prevention and early treatment of post-traumatic stress disorder and acute stress disorder: a systematic review and meta-analysis. Transl Psychiatry. 2019;9(1):334. 
3. Cirillo P, Gold AK, Nardi AE, Ornelas AC, Nierenberg AA, Camprodon J. \& Kinrys G.Transcranial magnetic stimulation in anxiety and traumarelated disorders: A systematic review and meta-analysis. Brain Behaviour. 2019;9(6):e01284.

4. Blumberger DM, Vila-Rodriguez F, Thorpe KE, Feffer K, Noda Y, Giacobbe $P$, et al. Effectiveness of theta burst versus high-frequency repetitive transcranial magnetic stimulation in patients with depression (THREED): a randomised non-inferiority trial. Lancet (London, England). 2018:391(10131):1683-1692.

5. Philip NS, Barredo J, Aiken E, Larson V, Jones RN, Shea MT, et al. ThetaBurst Transcranial Magnetic Stimulation for Posttraumatic Stress Disorder. Am J Psychiatry. 2019;176(11):939-48.

6. Nursey J, Sbisa A, Knight H, Ralph N, Cowlishaw S, Forbes D, et al. Exploring Theta Burst Stimulation for Post-traumatic Stress Disorder in Australian Veterans-A Pilot Study. Military Medicine. 2020;185(9-10):e1770-8.

7. Philip NS, Doherty RA, Faucher C, Aiken E, van't Wout-Frank M. Transcranial Magnetic Stimulation for Posttraumatic Stress Disorder and Major Depression: Comparing Commonly Used Clinical Protocols. J Traumatic Stress. 2021;0:1-8.

8. Kan RL, Zhang BB, Zhang JJ, Kranz GS. Non-invasive brain stimulation for posttraumatic stress disorder: a systematic review and meta-analysis. Translational Psychiatry. 2020;10(1):1-2.

9. Berlim MT, Van den Eynde F, Daskalakis ZJ. A systematic review and meta-analysis on the efficacy and acceptability of bilateral repetitive transcranial magnetic stimulation (rTMS) for treating major depression. Psychol Med. 2013;43(11):2245-54.

10. Berlim M, Van den Eynde F, Daskalakis ZJ. Clinically Meaningful Efficacy and Acceptability of Low-Frequency Repetitive Transcranial Magnetic Stimulation (rTMS) for Treating Primary Major Depression: A Meta-Analysis of Randomized, Double-Blind and Sham-Controlled Trials. Neuropsychopharmacol. 2013:38:543-51.

11. Palmiero M, Piccardi L. Frontal EEG asymmetry of mood: A mini-review. Front Behav Neurosci. 2017;6:224.

12. Bruder GE, Stewart JW, McGrath PJ. Right brain, left brain in depressive disorders: clinical and theoretical implications of behavioral, electrophysiological and neuroimaging findings. Neurosci Biobehavioral Reviews. 2017;1:178-91.

13. Blumberger DM, Maller JJ, Thomson L, Mulsant BH, Rajij TK, Maher M, Brown PE, Downar J, Vila-Rodriguez F, Fitzgerald PB, Daskalakis ZJ. Unilateral and bilateral MRI-targeted repetitive transcranial magnetic stimulation for treatment-resistant depression: a randomized controlled study. J Psychiatry Neurosci. 2016;41(4):E58.

14. Trevizol AP, Goldberger KW, Mulsant BH, Rajji TK, Downar J, Daskalakis ZJ, Blumberger DM. Unilateral and bilateral repetitive transcranial magnetic stimulation for treatment-resistant late-life depression. Int J Geriatric Psychiatry. 2019;34(6):822-7.

15. Plewnia C, Pasqualetti P, Große S, Schlipf S, Wasserka B, Zwissler B, et al. Treatment of major depression with bilateral theta burst stimulation: a randomized controlled pilot trial. J Affect Disord. 2014;156:219-23.

16. Burhan AM, Patience JA, Teselink J, Marlatt NM, Babapoor-Farrokhran S, Palaniyappan L. Bilateral sequential theta burst stimulation for multipletherapy-resistant depression: A naturalistic observation study. J Psychiatric Res. 2020;130:342-6.

17. Baeken C, De Raedt R, Van Schuerbeek P, Vanderhasselt MA, De Mey J, Bossuyt A, et al. Right prefrontal HF-rTMS attenuates right amygdala processing of negatively valenced emotional stimuli in healthy females. Behavioural Brain Res. 2010;214(2):450-5.

18. Duprat R, Desmyter S, Rudi D, van Heeringen K, Van den Abbeele D, Tandt $\mathrm{H}$, et al. Accelerated intermittent theta burst stimulation treatment in medication-resistant major depression: A fast road to remission? J Affective Disorders. 2016;200:6-14.

19. Leucht S, Fennema H, Engel R, Kaspers-Janssen M, Lepping P, Szegedi A. What does the HAMD mean? J. Affect. Disord. 2013;148(2-3):243-248.

20. Weathers FW, Litz BT, Keane TM, Palmieri PA, Marx BP, \& Schnurr PP. The PTSD Checklist for DSM-5 (PCL-5) - Standard [Measurement instrument]. 2013. Available from https://www.ptsd.va.gov/.

21. Kroenke K, Spitzer RL, Williams JB. The PHQ-9: Validity of a brief depression severity measure. J Gen Intern Med. 2001;16(9):606-613.

22. Spitzer RL, Kroenke K, Williams JBW, Lowe B. A brief measure for assessing generalized anxiety disorder: the GAD-7. Arch Intern Med. 2006;166(10):1092-1097.
23. Beam W, Borckardt JJ, Reeves ST, George MS. An efficient and accurate new method for locating the F3 position for prefrontal TMS applications. Brain Stimulation. 2009;2(1):50-4.

24. McGirr A, Devoe DJ, Raedler A, Debert CT, Ismail Z, Berlim MT. Repetitive Transcranial Magnetic Stimulation for the Treatment of Post-traumatic Stress Disorder: A Systematic Review and Network Meta-analysis: La Stimulation Magnétique Transcrânienne Répétitive Pour le Traitement du Trouble de Stress Post-Traumatique : Une Revue Systématique et une Méta-Analyse en Réseau. Can J Psychiatry. 2021;66(9):763-73. https://doi. org/10.1177/0706743720982432. Epub 2020 Dec 23.

\section{Publisher's Note}

Springer Nature remains neutral with regard to jurisdictional claims in published maps and institutional affiliations.

Ready to submit your research? Choose BMC and benefit from:

- fast, convenient online submission

- thorough peer review by experienced researchers in your field

- rapid publication on acceptance

- support for research data, including large and complex data types

- gold Open Access which fosters wider collaboration and increased citations

- maximum visibility for your research: over $100 \mathrm{M}$ website views per year

At BMC, research is always in progress.

Learn more biomedcentral.com/submissions 